\title{
Strict Left (Right)-Conjunctive Left (Right) Semi-Uninorms and Implications Satisfying the Order Property
}

\author{
Zhudeng Wang ${ }^{1, ~ *, ~ Y u a n ~ W a n g ~}{ }^{2}$, Keming Tang ${ }^{2}$ \\ ${ }^{1}$ School of Mathematics and Statistics, Yancheng Teachers University, Yancheng, People's Republic of China \\ ${ }^{2}$ College of Information Engineering, Yancheng Teachers University, Yancheng, People's Republic of China
}

Email address:

zhudengwang2004@163.com (Zhudeng Wang), yctuwangyuan@163.com (Yuan Wang), tkmchina@126.com (Keming Tang)

${ }^{*}$ Corresponding author

\section{To cite this article:}

Zhudeng Wang, Yuan Wang, Keming Tang. Strict Left (Right)-Conjunctive Left (Right) Semi-Uninorms and Implications Satisfying the Order Property. Applied and Computational Mathematics. Vol. 6, No. 1, 2017, pp. 45-53. doi: 10.11648/j.acm.20170601.13

Received: January 8, 2017; Accepted: January 19, 2017; Published: February 23, 2017

\begin{abstract}
We firstly give out the formulas for calculating the upper and lower approximation strict left (right)-conjunctive left (right) semi-uninorms of a binary operation. Then, we lay bare the formulas for calculating the upper and lower approximation implications, which satisfy the order property, of a binary operation. Finally, we reveal the relationships between the upper approximation strict left (right)-conjunctive left (right) arbitrary $\vee$-distributive left (right) semi-uninorms and lower approximation right arbitrary $\wedge$-distributive implications which satisfy the order property.
\end{abstract}

Keywords: Fuzzy Logic, Fuzzy Connective, Left (Right) Semi-Uninorm, Implication, Strict Left (Right)-Conjunctive

\section{Introduction}

In fuzzy logic systems (see [1-2]), connectives "and", "or" and "not" are usually modeled by $t$-norms, $t$-conorms, and strong negations on $[0,1]$ (see [3]), respectively. Based on these logical operators on $[0,1]$, the three fundamental classes of fuzzy implications on $[0,1]$, i.e., $R$-, $S$-, and $Q L$-implications on $[0,1]$, were defined and extensively studied. But, as was pointed out by Fodor and Keresztfalvi [4], sometimes there is no need of the commutativity or associativity for the connectives "and" and "or". Thus, many authors investigated implications based on some other operators like weak $t$-norms [5], pseudo $t$-norms [6], pseudo-uninorms [7], left and right uninorms [8], semi-uninorms [9], aggregation operators [10] and so on.

Uninorms, introduced by Yager and Rybalov [11], and studied by Fodor et al. [12], are special aggregation operators that have proven useful in many fields like fuzzy logic, expert systems, neural networks, aggregation, and fuzzy system modeling. This kind of operation is an important generalization of both $t$-norms and $t$-conorms and a special combination of $t$-norms and $t$-conorms. But, there are real-life situations when truth functions cannot be associative or commutative. By throwing away the commutativity from the axioms of uninorms, Mas et al. introduced the concepts of left and right uninorms on $[0,1]$ in [13] and later in a finite chain in [14], and Wang and Fang [8, 15] studied the left and right uninorms on a complete lattice. By removing the associativity and commutativity from the axioms of uninorms, Liu [9] introduced the concept of semi-uninorms, and Su et al. [16] discussed the notions of left and right semi-uninorms, on a complete lattice. On the other hand, it is well known that a uninorm (semi-uninorm) $U$ can be conjunctive or disjunctive whenever $U(0,1)=0$ or 1 , respectively. This fact allows us to use uninorms in defining fuzzy implications.

Constructing fuzzy connectives is an interesting topic. Recently, Jenei and Montagna [17] introduced several new types of constructions of left-continuous $t$-norms, Wang [18] laid bare the formulas for calculating the smallest pseudo- $t$-norm that is stronger than a binary operation and the largest implication that is weaker than a binary operation, $\mathrm{Su}$ et al. [16] studied the constructions of left and right semi-uninorms on a complete lattice, $\mathrm{Su}$ and Wang [19] investigated the constructions of implications and coimplications on a complete lattice. and Wang et al. [20-22] studied the relations among implications, coimplications and left (right) semi-uninorms, on a complete lattice. Moreover, Wang et al. [23-24] investigated the constructions of conjunctive left (right) semi-uninorms, disjunctive left (right) 
semi-uninorms, strict left (right)-disjunctive left (right) semi-uninorm, implications and coimplications satisfying the neutrality principle.

This paper is a continuation of [16, 19, 23-24]. Motivated by these works in $[16,19,23-24]$, we will further focus on this issue and investigate constructions of the upper and lower approximation strict left (right)-conjunctive left (right) semi-uninorms and the upper and lower approximation implications which satisfy the order property.

This paper is organized as follows. In Section 2, we give out the formulas for calculating the upper and lower approximation strict left (right)-conjunctive left (right) semi-uninorms of a binary operation. In Section 3, we lay bare the formulas for calculating the upper and lower approximation implications, which satisfy the order property, of a binary operation. In Section 4, we reveal the relationships between the upper approximation strict left (right)-conjunctive left (right) arbitrary $\vee$-distributive left (right) semi-uninorms and lower approximation right arbitrary $\wedge$-distributive implications which satisfy the order property, and find out some conditions such that the lower approximation strict left (right)-conjunctive left (right) semi-uninorm of a binary operation and upper approximation implication, which satisfies the order property, of left (right) residuum of the binary operation satisfy the generalized modus ponens rule.

The knowledge about lattices required in this paper can be found in [25].

Throughout this paper, unless otherwise stated, $L$ always represents any given complete lattice with maximal element 1 and minimal element $0 ; J$ stands for any index set.

\section{Strict Conjunctive Left and Right Semi-Uninorms}

In this section, we firstly recall some necessary concepts about the strict conjunctive left (right) semi-uninorms on a complete lattice.

Definition 2.1 (Su et al. [16]). A binary operation $U$ on $L$ is called a left (right) semi-uninorm if it satisfies the following two conditions:

(U1) there exists a left (right) neutral element, i.e., an element $e_{L} \in L \quad\left(e_{R} \in L\right)$ satisfying $U\left(e_{L}, x\right)=x$ $\left(U\left(x, e_{R}\right)=x\right.$ for all $x \in L$,

(U2) $U$ is non-decreasing in each variable.

Clearly, $U(0,0)=0$ and $U(1,1)=1$ hold for any left (right) semi-uninorm $U$ on $L$.

If a left (right) semi-uninorm $U$ is associative, then $U$ is the left (right) uninorm [8, 15] on $L$.

If a left (right) semi-uninorm $U$ with the left (right) neutral element $e_{L} \in L \quad\left(e_{R} \in L\right)$ has a right (left) neutral element $e_{R} \in L \quad\left(e_{L} \in L\right)$, then $e_{L}=U\left(e_{L}, e_{R}\right)=e_{R}$. Let $e=e_{L}=e_{R}$. Here, $U$ is the semi-uninorm [9].

For any left (right) semi-uninorm $U$ on $L, U$ is said to be left-conjunctive and right-conjunctive if $U(0,1)=0$ and
$U(1,0)=0$, respectively. $U$ is said to be conjunctive if both $U(1,0)=0$ and $U(1,0)=0$ since it satisfies the classical boundary conditions of AND.

$U$ is said to be strict left-conjunctive and strict rightconjunctive if $U$ is conjunctive and for any $x \in L, U(x, 1)=0 \Leftrightarrow x=0 \quad$ and $\quad U(1, x)=0 \Leftrightarrow x=0$, respectively.

Definition 2.2 (Wang and Fang [8]). A binary operation $U$ on $L$ is called left (right) arbitrary $\vee$-distributive if

$$
\begin{array}{r}
U\left(\vee_{j \in J} x_{j}, y\right)=\vee_{j \in J} U\left(x_{j}, y\right) \quad \forall x_{j}, y \in L \\
\left(U\left(x, \vee_{j \in J} y_{j}\right)=\vee_{j \in J} U\left(x, y_{j}\right) \quad \forall x, y_{j} \in L\right) ;
\end{array}
$$

left (right) arbitrary $\wedge$-distributive if

$$
\begin{gathered}
U\left(\wedge_{j \in J} x_{j}, y\right)=\wedge_{j \in J} U\left(x_{j}, y\right) \quad \forall x_{j}, y \in L \\
\left(U\left(x, \wedge_{j \in J} y_{j}\right)=\wedge_{j \in J} U\left(x, y_{j}\right) \quad \forall x, y_{j} \in L\right) .
\end{gathered}
$$

If a binary operation $U$ is left arbitrary $\vee$-distributive $(\wedge$ distributive) and also right arbitrary $\vee$-distributive $(\wedge$ -distributive), then $U$ is said to be arbitrary $\vee$-distributive ( $\wedge$-distributive).

For the sake of convenience, we introduce the following symbols:

$U_{c s}^{s e_{L}}(L)$ : the set of all strict left-conjunctive left semi-uninorms with the left neutral element $e_{L}$ on $L$;

$U_{c s}^{e_{R} s}(L)$ : the set of all strict right-conjunctive right semi-uninorms with the right neutral element $e_{R}$ on $L$;

$U_{v c s}^{s e_{L}}(L)$ : the set of all strict left-conjunctive left arbitrary $\checkmark$-distributive left semi-uninorms with the left neutral element $e_{L}$ on $L$;

$U_{c s \vee}^{e_{R} s}(L)$ : the set of all strict right-conjunctive right arbitrary $\checkmark$-distributive right semi-uninorms with the right neutral element $e_{R}$ on $L$.

Below, we illustrate these notions by means of two examples.

Example 2.1. Let $e_{L} \in L$,

$$
\begin{aligned}
& U_{c S M}^{e_{L}}(x, y)=\left\{\begin{array}{rr}
0 & \text { if } x=0 \text { or } y=0 \\
y & \text { if } 0<x \leq e_{L}, y \neq 0 \\
1 & \text { otherwise }
\end{array}\right. \\
& U_{c s W}^{s e_{L}}(x, y)=\left\{\begin{array}{cc}
y & \text { if } x \geq e_{L}, \\
\wedge\{a \in L \mid a \neq 0\} & \text { if } 0<x \text { not } \geq e_{L}, y=1, \\
0 & \text { otherwise. }
\end{array}\right.
\end{aligned}
$$

where $x$ and $y$ are elements of $L$. By Example 2 and Theorem 8 in [20], we see that $U_{c s}^{s e_{L}}(L)$ and $U_{v c s}^{s e_{L}}(L)$ are two join-semilattices with the greatest element $U_{c s M}^{e_{L}}$. When 
$e_{L} \neq 0$ and $\wedge\{a \in L \mid a \neq 0\} \neq 0$, it is straightforward to verify that $U_{c s W}^{s e_{L}}$ is the smallest element of $U_{c s}^{s e_{L}}(L)$.

Moreover, assume that $\vee\left\{a \in L \mid a\right.$ not $\left.\geq e_{L}\right\}$ not $\geq e_{L}$. $U_{c s W}^{s e_{L}}$ is left arbitrary $\vee$-distributive and the smallest element of $U_{\mathrm{v} c s}^{s e_{L}}(L)$.

Example 2.2. Let $e_{R} \in L$,

$$
\begin{gathered}
U_{c s M}^{e_{R}}(x, y)=\left\{\begin{array}{cc}
0 & \text { if } x=0 \text { or } y=0, \\
x & \text { if } 0<y \leq e_{R}, x \neq 0, \\
1 & \text { otherwise, }
\end{array}\right. \\
U_{c s W}^{e_{R} s}(x, y)=\left\{\begin{array}{cc}
x & \text { if } y \geq e_{R}, \\
\wedge\{a \in L \mid a \neq 0\} & \text { if } 0<y \text { not } \geq e_{R}, x=1, \\
0 & \text { otherwise. }
\end{array}\right.
\end{gathered}
$$

where $x$ and $y$ are elements of $L$. By Example 3 and Theorem 8 in [20], we see that $U_{c s}^{e_{R} s}(L)$ and $U_{c s v}^{e_{R} s}(L)$ are two join-semilattices with the greatest element $U_{c S M}^{e_{R}}$.

Similarly, When $e_{L} \neq 0$ and $\wedge\{a \in L \mid a \neq 0\} \neq 0, U_{c s W}^{e_{R} s}$ is the smallest element of $U_{c s}^{e_{R} s}(L)$. Moreover, if $\vee\left\{a \in L \mid a \operatorname{not} \geq e_{R}\right\}$ not $\geq e_{R}$, then $U_{c s W}^{e_{R} s}$ is the smallest element of $U_{c s \vee}^{e_{R} s}(L)$.

Constructing aggregation operators is an interesting work. Recently, Jenei and Montagna [17] introduced several new types of constructions of left-continuous $t$-norms, Su et al. [16] studied the constructions of left and right semi-uninorms on a complete lattice, and Wang et al. [23-24] investigated the constructions of conjunctive left (right) semi-uninorms and disjunctive left (right) semi-uninorms on a complete lattice. Now, we continue this work and give out the formulas for calculating the upper and lower approximation strict left (right)-conjunctive left (right) semi-uninorms of a binary operation.

It is easy to verify that $\vee_{j \in J} U_{j} \in U_{c s}^{s e_{L}}(L)$ for any nonempty subset $\left\{U_{j} \mid j \in J\right\}$ of $U_{c s}^{s e_{L}}(L)$. If $e_{L} \neq 0$ and $\wedge\{a \in L \mid a \neq 0\} \neq 0$, then $U_{c s}^{s e_{L}}(L)$ is a complete lattice with the smallest element $U_{c s W}^{s e_{L}}$ and greatest element $U_{c s M}^{e_{L}}$ by Example 2.1. Thus, for a binary operation $A$ on $L$, if there exists $U \in U_{c s}^{s e_{L}}(L)$ such that $A \leq U$, then

$$
\wedge\left\{U \mid A \leq U, U \in U_{c s}^{s e_{L}}(L)\right\}
$$

is the smallest strict left-conjunctive left semi-uninorm that is stronger than $A$ on $L$, we call it the upper approximation strict left-conjunctive left semi-uninorm of $A$ and write as $[A)_{c s}^{s e_{L}}$; if there exists $U \in U_{c s}^{s e_{L}}(L)$ such that $U \leq A$, then

$$
\vee\left\{U \mid U \leq A, U \in U_{c s}^{s e_{L}}(L)\right\}
$$

is the largest strict left-conjunctive left semi-uninorm that is weaker than $A$ on $L$, we call it the lower approximation strict left-conjunctive left semi-uninorm of $A$ and write as $(A]_{C S}^{s e_{L}}$.

Similarly, we introduce the following symbols:

$[A)_{c s}^{e_{R} s}$ : the upper approximation strict right-conjunctive right semi-uninorm of $A$;

$(A]_{c s}^{e_{R} s}$ : the lower approximation strict right-conjunctive right semi-uninorm of $A$;

$[A)_{v c s}^{s e_{L}}$ : the upper approximation strict left-conjunctive left arbitrary $\vee$-distributive left semi-uninorm of $A$;

$(A]_{\vee c S}^{s e_{L}}$ : the lower approximation strict left-conjunctive left arbitrary $\vee$-distributive left semi-uninorm of $A$;

$[A)_{c s v}^{e_{R} s}:$ the upper approximation strict right-conjunctive right arbitrary $\vee$-distributive right semi-uninorm of $A$;

$(A]_{c s}^{e_{R} s}$ : the lower approximation strict right-conjunctive right arbitrary $\vee$-distributive right semi-uninorm of $A$.

Definition 2.3 (Su et al. [16]). Let $A$ be a binary operation on $L$. Define the upper approximation aggregator $A_{u a}$ and the lower approximation aggregator $A_{l a}$ of $A$ as follows:

$$
\begin{aligned}
& A_{u a}(x, y)=\vee\{A(u, v) \mid u \leq x, v \leq y\} \quad \forall x, y \in L, \\
& A_{l a}(x, y)=\wedge\{A(u, v) \mid u \geq x, v \geq y\} \quad \forall x, y \in L .
\end{aligned}
$$

Theorem 2.1 (Su et al. [16]). Let $A, B \in L^{L \times L}$. Then the following statements hold:

$$
\begin{gathered}
A_{l a} \leq A \leq A_{u a} \\
(A \vee B)_{u a}=A_{u a} \vee B_{u a} \text { and } \\
(A \wedge B)_{l a}=A_{l a} \wedge B_{l a} .
\end{gathered}
$$

$A_{u a}$ and $A_{l a}$ are non-decreasing in its each variable.

If $A$ is non-decreasing in its each variable, then

$$
A_{u a}=A_{l a}=A \text {. }
$$

Theorem 2.2. Let $A \in L^{L \times L}$.

(1) If $A$ is left (right) arbitrary $\vee$-distributive, then $A_{u a}$ is left (right) arbitrary $\vee$-distributive.

(2) If $A$ is left (right) arbitrary $\wedge$-distributive, then

$$
A_{l a} \text { is left (right) arbitrary } \wedge \text {-distributive. }
$$

Proof. We only prove that statement (1) holds.

If $A$ is left arbitrary $\vee$-distributive, then $A$ is non-decreasing in its first variable,

$$
\begin{aligned}
& A_{u a}(x, y)=\vee\{A(u, v) \mid u \leq x, v \leq y\} \\
& =\vee\{A(x, v) \mid v \leq y\} \quad \forall x, y \in L,
\end{aligned}
$$




$$
\begin{aligned}
& A_{u a}\left(\vee_{j \in J} x_{j}, y\right)=\vee\left\{A\left(\vee_{j \in J} x_{j}, v\right) \mid v \leq y\right\} \\
& =\vee\left\{\vee_{j \in J} A\left(x_{j}, v\right) \mid v \leq y\right\}=\vee_{j \in J}\left\{\vee A\left(x_{j}, v\right) \mid v \leq y\right\} \\
& =\vee_{j \in J} A_{u a}\left(x_{j}, y\right) \quad \forall x_{j}, y \in L(j \in J),
\end{aligned}
$$

i.e., $A_{u a}$ is left arbitrary $\vee$-distributive.

Similarly, we can show that $A_{u a}$ is right arbitrary $\vee$ -distributive when $A$ is right arbitrary $\vee$-distributive.

The theorem is proved.

Below, we give out the formulas for calculating the upper and lower approximation strict left (right)-conjunctive left (right) semi-uninorms of a binary operation.

Theorem 2.3. Suppose that $A \in L^{L \times L}, e_{L} \neq 0$ and $\wedge\{a \in L \mid a \neq 0\} \neq 0$.

(1) If $A \leq U_{c s M}^{e_{L}}$, then $[A)_{c s}^{s e_{L}}=U_{c s W}^{s e_{L}} \vee A_{u a}$;

$$
\text { if } U_{c s W}^{s e_{L}} \leq A \text {, then }(A]_{c s}^{s e_{L}}=U_{c s M}^{e_{L}} \wedge A_{l a} \text {. }
$$

(2) If $\vee\left\{a \in L \mid a\right.$ not $\left.\geq e_{L}\right\}$ not $\geq e_{L}, \quad A \leq U_{c s M}^{e_{L}}$ and $A$ is left arbitrary $\vee$-distributive, then

$$
[A)_{\vee c s}^{s e_{L}}=U_{c s W}^{s e_{L}} \vee A_{u a} .
$$

Moreover, if $A$ is non-decreasing in its second variable, then $[A)_{\vee c s}^{s e_{L}}=U_{c s W}^{s e_{L}} \vee A$.

Proof. Assume that $e_{L} \neq 0$ and $\wedge\{a \in L \mid a \neq 0\} \neq 0$. Then $U_{c s W}^{s e_{L}}$ and $U_{c s M}^{e_{L}}$ are, respectively, the smallest and greatest elements of $U_{c s}^{s e_{L}}(L)$ by Example 2.1.

(1) Let $U_{1}=U_{c s W}^{s e_{L}} \vee A_{u a}$. If $A \leq U_{c s M}^{e_{L}}$, then $A \leq U_{1}$, $A_{u a} \leq\left(U_{c s M}^{e_{L}}\right)_{u a}=U_{c s M}^{e_{L}}$. Thus,

$$
U_{c s W}^{e_{L}} \leq U_{1} \leq U_{c s M}^{e_{L}}
$$

It implies that $U_{1}(1,0)=U_{1}(0,1)=0$ and $U_{1}\left(e_{L}, y\right)=y$ for any $y \in L$. If $U_{1}(x, 1)=0$, then $U_{c s W}^{s e_{L}}(x, 1)=0$ and so $x=0$, i.e., $U_{1}$ is strict left-conjunctive. By Theorem 2.1(3) and the monotonicity of $U_{c s W}^{s e_{L}}$, we can see that $U_{1}$ is non-decreasing in its each variable. So, $U_{1} \in U_{c s}^{s e_{L}}(L)$. If $A \leq U$ and $U \in U_{c s}^{s e_{L}}(L)$, then $A_{u a} \leq U_{u a}=U$ and $U_{1}=U_{c s W}^{s e_{L}} \vee A_{u a} \leq U$. Therefore,

$$
[A)_{c s}^{s e_{L}}=U_{c s W}^{s e_{L}} \vee A_{u a} .
$$

Let $U_{2}=U_{c s M}^{e_{L}} \wedge A_{l a}$. If $U_{c s W}^{s e_{L}} \leq A$, then

$$
U_{c s W}^{s e_{L}}=\left(U_{c s W}^{s e_{L}}\right)_{l a} \leq A_{l a} \text { and } U_{c s W}^{s e_{L}} \leq U_{2} \leq U_{c s M}^{e_{L}}
$$

Thus, $U_{2}(1,0)=U_{2}(0,1)=0$ and $U_{2}\left(e_{L}, y\right)=y$ for any $y \in L$ and $U_{2}$ is strict left-conjunctive. By Theorem 2.1(3) and the monotonicity of $U_{c s M}^{e_{L}}$, we know that $U_{2}$ is non-decreasing in its each variable. So, $U_{2} \in U_{c s}^{s e_{L}}(L)$. If
$U \leq A \quad$ and $\quad U \in U_{c s}^{s e_{L}}(L) \quad$, then $\quad U=U_{l a} \leq A_{l a} \quad$ and $U \leq U_{c s M}^{e_{L}} \wedge A_{l a}=U_{2}$. Therefore,

$$
(A]_{c S}^{S e_{L}}=U_{c s M}^{e_{L}} \wedge A_{l a}
$$

(2) When $\vee\left\{a \in L \mid a\right.$ not $\left.\geq e_{L}\right\}$ not $\geq e_{L}, U_{c s W}^{s e_{L}}$ and $U_{c s M}^{e_{L}}$ are, respectively, the smallest and greatest elements of $U_{v c s}^{s e_{L}}(L)$ by Example 2.1. Let $U_{3}=U_{c s W}^{s e_{L}} \vee A_{u a}$. If $A \leq U_{c s M}^{e_{L}}$, then $U_{3} \in U_{c s}^{s e_{L}}(L)$ by statement (1). Noting that $A$ is left arbitrary $\vee$-distributive, we can see that $A_{u a}$ is also left arbitrary $\vee$-distributive by Theorem 2.2(1). Thus, $U_{3}$ is left arbitrary $\vee$-distributive and $U_{3} \in U_{\vee c S}^{s e_{L}}(L)$. By the proof of statement (1), we have that $[A)_{\vee c s}^{s e_{L}}=U_{c s W}^{s e_{L}} \vee A_{u a}$.

Moreover, if $A$ is non-decreasing in its second variable, then $A_{u a}=A$ by Theorem 2.1(4) and so

$$
[A)_{\vee c s}^{s e_{L}}=U_{c s W}^{s e_{L}} \vee A
$$

The theorem is proved.

Similarly, for calculating the upper and lower approximation strict right-conjunctive right semi-uninorms of a binary operation, we have the following theorem.

Theorem 2.4. Suppose that $A \in L^{L \times L}, e_{R} \neq 0$ and $\wedge\{a \in L \mid a \neq 0\} \neq 0$.

(1) If $A \leq U_{c s M}^{e_{R}}$, then $[A)_{c s}^{e_{R} s}=U_{c s W}^{e_{R} s} \vee A_{u a}$; if $U_{c s W}^{e_{R} s} \leq A$, then $(A]_{c s}^{e_{R} s}=U_{c s M}^{e_{R}} \wedge A_{l a}$.

(2) If $\vee\left\{a \in L \mid a\right.$ not $\left.\geq e_{R}\right\}$ not $\geq e_{R}, A \leq U_{c s M}^{e_{R}}$ and $A$ is right arbitrary $\vee$-distributive, then

$$
[A)_{c s \vee}^{e_{R} s}=U_{c s W}^{e_{R} s} \vee A_{u a} .
$$

Moreover, if $A$ is non-decreasing in its first variable, then $[A)_{c s \vee}^{e_{R} s}=U_{c s W}^{e_{R} s} \vee A$.

\section{Implications Satisfying the Order Property}

Recently, Su and Wang [19] have studied the constructions of implications and coimplications and Wang et al. [23-24] further investigated the constructions of implications and coimplications satisfying the neutrality principle on a complete lattice. This section is a continuation of [19, 23-24]. We will study the constructions of the upper and lower approximation implications which satisfy the order property.

Definition 3.1 (Baczynski and Jayaram [26], Bustince et al. [27], De Baets and Fodor [28], Fodor and Roubens [1]). An implication $I$ on $L$ is a hybrid monotonous (with decreasing first and increasing second partial mappings) binary operation that satisfies the corner conditions $I(0,0)=I(1,1)=1$ and $I(1,0)=0$.

An implication $I$ is said to satisfy the order property with respect to $e$ (w.r.t. e, for short) when $x \leq y$ if and 
only if $I(x, y) \geq e$ for any $x, y \in L$.

Note that for any implication $I$ on $L$, due to the monotonicity, the absorption principle holds, i.e., $I(0, x)=I(x, 1)=1$ for any $x \in L$.

For the sake of convenience, we introduce the following symbols:

$I^{\text {ope }}(L)$ : the set of all implications which satisfy the order property w. r. t. $e$ on $L$;

$I_{\wedge}^{\text {ope }}(L)$ : the set of all right arbitrary $\wedge$-distributive implications which satisfy the order property w. r. t. $e$ on $L$.

Clearly, $I^{\text {ope }}(L)$ and $I_{\wedge}^{\text {ope }}(L)$ are all meet-semilattices. Definition 3.2. Let $U$ be a binary operation on $L$. Define $I_{U}^{L}, I_{U}^{R} \in L^{L \times L}$ as follows:

$$
\begin{aligned}
& I_{U}^{L}(x, y)=\vee\{z \in L \mid U(z, x) \leq y\} \quad \forall x, y \in L, \\
& I_{U}^{R}(x, y)=\vee\{z \in L \mid U(x, z) \leq y\} \quad \forall x, y \in L .
\end{aligned}
$$

Here, $I_{U}^{L}$ and $I_{U}^{R}$ are, respectively, called the left and right residuum of the binary operation $U$.

When $U$ is non-decreasing in each variable, it is easy to see that $I_{U}^{L}$ and $I_{U}^{R}$ are all decreasing in the first variable and increasing in the second one by Definition 3.2.

Example 3.1. For some left and right semi-uninorms in Examples 2.1-2.2, a simple computation shows that

$$
\begin{aligned}
& I_{U_{c s w}^{s e L}}^{L}(x, y)=\left\{\begin{array}{cr}
0 & \text { if } x=1 \text { and } y=0, \\
1 & \text { if } x \leq y, \\
\vee\left\{a \in L \mid a \text { not } \geq e_{L}\right\} & \text { otherwise, }
\end{array}\right. \\
& I_{U_{c S M}^{e_{M}}}^{L}(x, y)=\left\{\begin{array}{c}
1 \text { if } x=0 \text { or } y=1 \\
e_{L} \text { if } 0<x \leq y<1 \\
0 \quad \text { otherwise }
\end{array}\right. \\
& I_{U_{c s W}^{e_{S} S}}^{R}(x, y)=\left\{\begin{array}{lr}
0 & \text { if } x=1 \text { and } y=0, \\
1 & \text { if } x \leq y, \\
\vee\left\{a \in L \mid a \text { not } \geq e_{R}\right\} & \text { otherwise, }
\end{array}\right. \\
& I_{U_{c s M}^{e_{R}}}^{R}(x, y)=\left\{\begin{array}{cr}
1 & \text { if } x=0 \text { or } y=1 \\
e_{R} & \text { if } 0<x \leq y<1 \\
0 & \text { otherwise }
\end{array}\right.
\end{aligned}
$$

where $x$ and $y$ are elements of $L$. By the virtue of Theorem 8 in [20], we see that $I_{U_{c M}^{e_{L}}}^{L}$ is the smallest element of both $I^{o p e_{L}}(L)$ and $I_{\wedge}^{o p e_{L}}(L)$.

When $e_{L} \neq 0$ and $\vee\left\{a \in L \mid a\right.$ not $\left.\geq e_{L}\right\}$ not $\geq e_{L}$, it is easy to see that $I_{U_{c s W}^{s e L}}^{L}$ is the greatest element of $I^{o p e_{L}}(L)$.

Moreover, assume that $\wedge\{a \in L \mid a \neq 0\} \neq 0 . I_{U_{c s W}^{s e_{L}}}^{L}$ is the greatest element of $I_{\wedge}^{o p e_{L}}(L)$.
Similar conclusions hold for $I^{o p e_{R}}(L)$ and $I_{\wedge}^{o p e_{R}}(L)$.

It is easy to verify that if $J \neq \Phi$, then

$$
I_{j} \in I^{o p e_{L}}(L) \forall j \in J \Rightarrow \wedge_{j \in J} I_{j} \in I^{o p e_{L}}(L) .
$$

When $e_{L} \neq 0$ and $\vee\left\{a \in L \mid a\right.$ not $\left.\geq e_{L}\right\}$ not $\geq e_{L}$, we see that $I^{o p e_{L}}(L)$ is also a complete lattice with the smallest element $I_{U_{c s M}^{e L}}^{L}$ and greatest element $I_{U_{c s W}^{s e l}}^{L}$ by Example 3.1 . Thus, for a binary operation $A$ on $L$, if there exists $I \in I^{o p e_{L}}(L)$ such that $A \leq I$, then

$$
\wedge\left\{I \mid A \leq I, I \in I^{\text {ope }_{L}}(L)\right\}
$$

is the smallest implication that is stronger than $A$ and satisfies the order property w. r. t. $e_{L}$ on $L$. Here, we call it the upper approximation implication, which satisfies the order property w. r. t. $e_{L}$, of $A$ and write as $[A)_{I}^{o p e_{L}}$. Similarly, if there exists $I \in I^{\text {ope }_{L}}(L)$ such that $I \leq A$, then

$$
\vee\left\{I \mid I \leq A, I \in I^{o p e_{L}}(L)\right\}
$$

is the largest implication that is weaker than $A$ and satisfies the order property w. r. t. $e_{L}$ on $L$. Here, we call it the lower approximation implication, which satisfies the order property w. r. t. $e_{L}$, of $A$ and write as $(A]_{I}^{o p e_{L}}$.

Likewise, for a binary operation $A$ on $L$, we may introduce the following symbols:

$[A)_{I}^{\text {ope }_{R}}$ : the upper approximation implication, which satisfies the order property w. r. t. $e_{R}$, of $A$;

$(A]_{I}^{o p e_{R}}$ : the lower approximation implication, which satisfies the order property w. r. t. $e_{R}$, of $A$;

$[A)_{I}^{o p e_{L} \wedge}\left([A)_{I}^{o p e_{R} \wedge}\right)$ : the upper approximation right arbitrary $\wedge$-distributive implication, which satisfies the order property w. r. t. $e_{L}\left(e_{R}\right)$, of $A$;

$(A]_{I}^{o p e_{L} \wedge}\left((A]_{I}^{o p e_{R} \wedge}\right)$ : the lower approximation right arbitrary $\wedge$-distributive implication, which satisfies the order property w.r. t. $e_{L}\left(e_{R}\right)$, of $A$.

Definition 3.3 (see Su and Wang [19]). Let $A$ be a binary operation on $L$. Define the upper approximation implicator $A_{u i}$ and the lower approximation implicator $A_{l i}$ of $A$ as follows:

$$
\begin{array}{ll}
A_{u i}(x, y)=\vee\{A(u, v) \mid u \geq x, v \leq y\} & \forall x, y \in L, \\
A_{l i}(x, y)=\wedge\{A(u, v) \mid u \leq x, v \geq y\} & \forall x, y \in L .
\end{array}
$$

Theorem 3.1 (see $\mathrm{Su}$ and Wang [19]). Let $A, B \in L^{L \times L}$. Then the following statements hold:

$$
A_{l i} \leq A \leq A_{u i}
$$

$$
(A \vee B)_{u i}=A_{u i} \vee B_{u i} \text { and }
$$




$$
(A \wedge B)_{l i}=A_{l i} \wedge B_{l i}
$$

$A_{u i}$ and $A_{l i}$ are hybrid monotonous.

If $A$ is are hybrid monotonous, then $A_{u i}=A_{l i}=A$.

Theorem 3.2. Let $A \in L^{L \times L}$.

(1) If $A$ is right arbitrary $\vee$-distributive, then $A_{u i}$ is also right arbitrary $\vee$-distributive,

$$
\begin{gathered}
\left(I_{A}^{R}\right)_{l i}=I_{A_{u a}}^{R},\left(I_{A}^{R}\right)_{u i} \leq I_{A_{l a}}^{R}, \\
A_{\text {ua }}\left(x,\left(I_{A}^{R}\right)_{l i}(x, y)\right) \leq y \quad \forall x, y \in L .
\end{gathered}
$$

(2) If $A$ is right arbitrary $\wedge$-distributive, then $A_{l i}$ is also right arbitrary $\wedge$-distributive.

(3) If $A$ is left arbitrary $\vee$-distributive, then,

$$
\begin{aligned}
& \left(I_{A}^{L}\right)_{l i}=I_{A_{u a}}^{L},\left(I_{A}^{L}\right)_{u i} \leq I_{A_{l a}}^{L}, \\
& A_{u a}\left(\left(I_{A}^{L}\right)_{l i}(x, y), x\right) \leq y \quad \forall x, y \in L .
\end{aligned}
$$

Proof. We only prove that statement (1) holds.

Assume that $A$ is a right arbitrary $\vee$-distributive binary operation on $L$. Clearly, $A_{u a}$ is also right arbitrary $\vee$ -distributive. By Definition 3.3, the monotonicity of $A$ and $I_{A}^{R}$, and the right residual principle, we have that

$$
\begin{aligned}
& I_{A_{u a}}^{R}(x, y)=\vee\left\{z \in L \mid A_{u a}(x, z) \leq y\right\} \\
&=\vee\{z \in L \mid \vee\{A(u, v) \mid u \leq x, v \leq z\} \leq y\} \\
&=\vee\{z \in L \mid \vee\{A(u, z) \mid u \leq x\} \leq y\} \\
&=\vee\{z \in L \mid A(u, z) \leq y \forall u \leq x\} \\
&=\vee\left\{z \in L \mid z \leq I_{A}^{R}(u, y) \forall u \leq x\right\} \\
&=\vee\left\{z \in L \mid z \leq \wedge_{u \leq x} I_{A}^{R}(u, y)\right\} \\
&=\wedge_{u \leq x} I_{A}^{R}(u, y) \forall x, y \in L, \\
&\left(I_{A}^{R}\right)_{l i}(x, y)=\wedge\left\{I_{A}^{R}(u, v) \mid u \leq x, v \geq y\right\} \\
&=\wedge\left\{I_{A}^{R}(u, y) \mid u \leq x\right\}=\wedge_{u \leq x} I_{A}^{R}(u, y) \forall x, y \in L .
\end{aligned}
$$

Thus, $\left(I_{A}^{R}\right)_{l i}=I_{A_{u a}}^{R}$. Similarly, we have that

$$
\begin{aligned}
& \left(I_{A}^{R}\right)_{u i}(x, y)=\vee\left\{I_{A}^{R}(u, y) \mid u \geq x\right\} \forall x, y \in L, \\
& \quad A_{l a}(x, z)=\wedge\left\{A\left(u_{1}, v\right) \mid u_{1} \geq x, v \geq z\right\} \\
& =\wedge\left\{A\left(u_{1}, v\right) \mid u_{1} \geq x\right\} \forall x, z \in L, \\
& \left(I_{A_{l a}}^{R}\right)(x, y) \\
& =\vee\left\{z \in L \mid \wedge\left\{A\left(u_{1}, z\right) \mid u_{1} \geq x\right\} \leq y\right\} \forall x, y \in L .
\end{aligned}
$$

If $u \geq x$, let $z=I_{A}^{R}(u, y)$, then

$$
\begin{aligned}
& A(u, z)=A(u, \vee\{c \in L \mid A(u, c) \leq y\}) \\
& =\vee\{A(u, c) \mid A(u, c) \leq y\} \leq y, \\
& \wedge\left\{A\left(u_{1}, z\right) \mid u_{1} \geq x\right\} \leq A(u, z) \leq y .
\end{aligned}
$$

So, $\quad\left(I_{A}^{R}\right)_{u i}(x, y) \leq\left(I_{A_{l a}}^{R}\right)(x, y)$ for any $x, y \in L$, i.e., $\left(I_{A}^{R}\right)_{u i} \leq I_{A_{l a}}^{R}$. Moreover, we know that $A_{u a}$ is right arbitrary $\checkmark$-distributive and hence

$$
\begin{aligned}
& A_{u a}\left(x,\left(I_{A}^{R}\right)_{l i}(x, y)\right)=A_{u a}\left(x, I_{A_{u a}}^{R}(x, y)\right) \\
& =A_{u a}\left(x, \vee\left\{z \in L \mid A_{u a}(x, z) \leq y\right\}\right) \\
& =\vee\left\{A_{u a}(x, z) \mid A_{u a}(x, z) \leq y\right\} \leq y \forall x, y \in L .
\end{aligned}
$$

The theorem is proved.

Below, we give out the formulas for calculating the upper and lower approximation implications which satisfy the order property.

Theorem 3.3. Suppose that $A \in L^{L \times L}, e_{L} \neq 0$ and $\vee\left\{a \in L \mid a\right.$ not $\left.\geq e_{L}\right\}$ not $\geq e_{L}$.

(1) If $A \leq I_{U_{c s W}^{s e W^{L}}}^{L}$, then $[A)_{I}^{o p e_{L}}=I_{U_{c s M}^{e_{L}}}^{L} \vee A_{u i}$;

if $A \geq I_{U_{c s M}^{e_{L}}}^{L_{L}}$, then $(A]_{I}^{o p e_{L}}=I_{U_{c s W}^{s e_{L}}}^{L} \wedge A_{l i}$.

(2) If $\wedge\{a \in L \mid a \neq 0\} \neq 0, \quad A \geq I_{U_{c s M}^{e_{L}}}^{L}$ and $A$ is right arbitrary $\wedge$-distributive, then

$$
(A]_{I}^{o p e_{L} \wedge}=I_{U_{c s W}^{s e}}^{L} \wedge A_{l i} .
$$

Moreover, if $A$ is non-decreasing in its first variable, then $(A]_{I}^{\text {ope }_{L} \wedge}=I_{U^{s e_{L}}}^{L} \wedge A$.

Proof. Assume that $\vee\left\{a \in L \mid a\right.$ not $\left.\geq e_{L}\right\}$ not $\geq e_{L}$ and $e_{L} \neq 0$. Then $I_{U_{c s M}^{e_{L}}}^{L}$ and $I_{U_{c s}^{s e_{L}}}^{L}$ are, respectively, the smallest and greatest elements of $I^{o p e_{L}}(L)$ by Example 3.1.

(1) If $A \leq I_{U_{c s W}^{s e L}}^{L}$, let $I_{1}=I_{U_{c s M}^{e L}}^{L} \vee A_{u i}$, then $A \leq I_{1}$ and

$$
I_{U_{c s M}^{e_{L}}}^{L} \leq I_{1} \leq I_{U_{c s W}^{s e_{L}}}^{L_{L}}
$$

Thus, $I_{1}(0,0)=I_{1}(1,1)=1$ and $I_{1}(1,0)=0$. If $x \leq y$, then $\quad I_{1}(x, y) \geq I_{U_{c s M}^{e L}}^{L}(x, y) \geq e_{L} \quad ; \quad$ if $\quad I_{1}(x, y) \geq e_{L} \quad$, then $I_{U_{c s W}^{s e_{L}}}^{L}(x, y) \geq I_{1}(x, y) \geq e_{L}$ and so $x \leq y$, i.e., $I_{1}$ satisfies the order property w. r. t. $e_{L}$. By Theorem 3.1(3) and the hybrid monotonicity of $I_{U_{c M}^{e_{L}}}^{L_{L}}$, we know that $I_{1}$ is hybrid monotonous. So, $I_{1} \in I^{o p e_{L}}(L)$. If $A \leq I$ and $I \in I^{\text {ope }_{L}}(L)$, then $A_{u i} \leq I_{u i}=I$ and $I_{1}=I_{U_{c s M}^{s e_{L}}}^{L} \vee A_{u i} \leq I$. Therefore,

$$
[A)_{I}^{o p e_{L}}=I_{U_{c s M}^{s e L}}^{L} \vee A_{u i} .
$$


If $A \geq I_{U_{c s M}^{e_{L}}}^{L}$, let $I_{2}=I_{U_{c s W}^{s e_{L}}}^{L} \wedge A_{l i}$, then $I_{2} \leq A$,

$$
A_{l i} \geq\left(I_{U_{c s M}^{e L}}^{L}\right)_{l i}=I_{U_{c s M}^{e L}}^{L}, I_{U_{c s M}^{e L}}^{L} \leq I_{2} \leq I_{U_{c s W}^{s e L}}^{L} .
$$

Thus, we can prove in an analogous way that $I_{2} \in I^{\text {ope }_{L}}(L)$ and $(A]_{I}^{o p e_{L}}=I_{U_{c s W}^{s e_{L}}}^{L} \wedge A_{l i}$.

(2) When $\wedge\{a \in L \mid a \neq 0\} \neq 0, \quad I_{U_{c s M}^{e_{L}}}^{L}$ and $I_{U_{c s}^{s e}}^{L}$ are, respectively, the smallest and greatest elements of $I_{\wedge}{ }^{o p e_{L}}(L)$ by Example 3.1. Let $I_{3}=I_{U_{c s W}^{s e L}}^{L} \wedge A_{l i}$. If $A \geq I_{U_{c s M}^{e_{L}}}^{L}$, then $I_{3} \in I^{o p e_{L}}(L)$ by statement (1). Noting that $A$ is right arbitrary $\wedge$-distributive, we can see that $A_{l i}$ is also right arbitrary $\wedge$-distributive by Theorem 3.2(2). So, $I_{3}$ is right arbitrary $\wedge$-distributive, i.e., $I_{3} \in I_{\wedge}^{o p e_{L}}(L)$. By the proof of statement (1), we know that $(A]_{I}^{o p e_{L} \wedge}=I_{U_{c s W}^{s e_{L}}}^{L} \wedge A$.

Moreover, if $A$ is non-decreasing in its first variable, then $A_{l i}=A$ by Theorem 3.1(4) and so

$$
(A]_{I}^{o p e_{L} \wedge}=I_{U_{c s W}^{s e_{W}}}^{L} \wedge A .
$$

The theorem is proved.

Analogous to Theorem 3.3, we have the following theorem.

Theorem 3.4. Suppose that $A \in L^{L \times L}, e_{R} \neq 0$ and $\vee\left\{a \in L \mid a \operatorname{not} \geq e_{R}\right\}$ not $\geq e_{R}$.

(1) If $A \leq I_{U_{c s W}^{e_{e s} s}}^{R}$, then $[A)_{I}^{\text {ope }_{R}}=I_{U_{c S M}^{e_{R}}}^{R} \vee A_{u i}$;

$$
\text { if } A \geq I_{U_{c s M}^{e_{R}}}^{R} \text {, then }(A]_{I}^{o p e_{R}}=I_{U_{c s W}^{e^{R} S}}^{R} \wedge A_{l i} .
$$

(2) If $\wedge\{a \in L \mid a \neq 0\} \neq 0, \quad A \geq I_{U_{c \wedge M}^{e_{R}}}^{e^{2}}$ and $A$ is right arbitrary $\wedge$-distributive, then

$$
(A]_{I}^{o p e_{R} \wedge}=I_{U_{c s W}^{e R^{s}}}^{R} \wedge A_{l i} .
$$

Moreover, if $A$ is non-decreasing in its first variable, then $(A]_{I}^{\text {ope }_{R} \wedge}=I_{U_{c s}^{e_{R}}}^{R} \wedge A$.

\section{The Relations Between Strict (Right)-Conjunctive Left (Right) Semi-Uninorms and Implications}

In this section, we reveal the relationships between the upper approximation strict left (right)-conjunctive left (right) arbitrary $\vee$-distributive left (right) semi-uninorms and lower approximation right arbitrary $\wedge$-distributive implications which satisfy the order property.

Theorem 4.1. Suppose that $A \in L^{L \times L}, e_{L}, e_{R} \neq 0$ and $\wedge\{a \in L \mid a \neq 0\} \neq 0$.

(1) If $\vee\left\{a \in L \mid a\right.$ not $\left.\geq e_{L}\right\}$ not $\geq e_{L}, \quad A \leq U_{c s M}^{e_{L}}$ and $A$ is left arbitrary $\vee$-distributive, then

$$
\left(I_{A}^{L}\right]_{I}^{o p e_{L} \wedge}=I_{[A)_{v \in s}^{s e_{L}}}^{L} .
$$

(2) If $\vee\left\{a \in L \mid a\right.$ not $\left.\geq e_{R}\right\}$ not $\geq e_{R}, \quad A \leq U_{c s M}^{e_{R}}$ and $A$ is right arbitrary $\vee$-distributive, then

$$
\left(I_{A}^{R}\right]_{I}^{o p e_{R} \wedge}=I_{[A)_{c s v}^{e_{R} s}}^{R} .
$$

Proof. We only prove the statement (1) holds.

Assume that $A \leq U_{c s M}^{e_{L}}$ and $A$ is left arbitrary $\vee$ -distributive. Then it follows from Theorem 4.6 in [8] and Definition 3.2 that $I_{U_{c s M}^{e_{L}}}^{L} \leq I_{A}^{L}$ and $I_{A}^{L}$ is right arbitrary $\wedge$ -distributive. Thus, $\quad\left(I_{A}^{L}\right]_{I}^{o p e_{L} \wedge}=I_{U_{c s W}^{s e_{L}}}^{L} \wedge\left(I_{A}^{L}\right)_{l i}$ by Theorem 3.3(2). Moreover, it follows from Theorems 2.2(1) and 2.3(2) and the left residual principle that

$$
\begin{aligned}
& I_{[A)_{\vee c s}^{s e_{L}}}^{L}(x, y)=\vee\left\{z \in L \mid[A)_{\vee c s}^{s e_{L}}(z, x) \leq y\right\} \\
& =\vee\left\{z \in L \mid\left(U_{c s W}^{s e_{L}} \vee A_{u a}\right)(z, x) \leq y\right\} \\
& =\vee\left\{z \in L \mid U_{c s W}^{s e_{L}}(z, x) \vee A_{u a}(z, x) \leq y\right\} \\
& =\vee\left\{z \in L \mid U_{c s W}^{s e_{L}}(z, x) \leq y, A_{u a}(z, x) \leq y\right\} \\
& =\vee\left\{z \in L \mid z \leq I_{U_{c s W}^{s L_{W}}}^{L}(x, y), z \leq I_{A_{u a}}^{L}(x, y)\right\} \\
& =\vee\left\{z \in L \mid z \leq I_{U_{c s W}^{s e L}}^{L s}(x, y) \wedge I_{A_{u a}}^{L}(x, y)\right\} \\
& =\left(I_{U_{c s W}^{s e_{L}}}^{L} \wedge I_{A_{u a}}^{L}\right)(x, y) \forall x, y \in L,
\end{aligned}
$$

i.e., $I_{[A)_{\cup c s}^{s e_{L}}}^{L}=I_{U_{c s W}^{s e_{1}}}^{L} \wedge I_{A_{u a}}^{L}$. By Theorem 3.2(3), we know that $\left(I_{A}^{L}\right)_{l i}=I_{A_{u a}}^{L}$. Therefore,

$$
\left(I_{A}^{L}\right]_{I}^{o p e_{L} \wedge}=I_{U_{c s W}^{s e e_{L}}}^{L} \wedge\left(I_{A}^{L}\right)_{l i}=I_{U_{c s W}^{s e L}}^{L} \wedge I_{A_{u a}}^{L}=I_{[A)_{v c s}^{e_{L}}}^{L}
$$

The theorem is proved.

Finally, we give out some conditions such that the lower approximation strict left (right)-conjunctive left (right) semi-uninorm of a binary operation and upper approximation implication, which satisfies the order property, of left (right) residuum of the binary operation satisfy the GMP rule.

Theorem 4.2. Suppose that $A \in L^{L \times L}, e_{L}, e_{R} \neq 0$ and $\wedge\{a \in L \mid a \neq 0\} \neq 0$.

(1) If $\vee\left\{a \in L \mid a\right.$ not $\left.\geq e_{L}\right\}$ not $\geq e_{L}, \quad U_{c s W}^{s e_{L}} \leq A$ and $A$ is non-decreasing in its second variable and left arbitrary $\vee$-distributive and $I_{A}^{L}$ and $e_{L}$ are comparable (see [25]) when $0<x \leq y<1$, then $(A]_{c s}^{s e_{L}}$ and $\left[I_{A}^{L}\right)_{I}^{o p e_{L}}$ satisfy the GMP rule in the form

$$
(A]_{c s}^{s e_{L}}\left(\left[I_{A}^{L}\right)_{I}^{o p e_{L}}(x, y), x\right) \leq y \forall x, y \in L .
$$

(2) If $\vee\left\{a \in L \mid a\right.$ not $\left.\geq e_{R}\right\}$ not $\geq e_{R}, \quad U_{c s W}^{e_{R} s} \leq A$ and $A$ is non-decreasing in its first variable and right arbitrary $\checkmark$-distributive and $I_{A}^{R}$ and $e_{R}$ are comparable (see [25]) when $0<x \leq y<1$, then $(A]_{c s}^{e_{R} s}$ and $\left[I_{A}^{R}\right)_{I}^{\text {ope } e_{R}}$ satisfy the GMP rule in the form 


$$
(A]_{c s}^{e_{R} s}\left(x,\left[I_{A}^{R}\right)_{I}^{o p e_{R}}(x, y)\right) \leq y \forall x, y \in L .
$$

Proof. We only prove the statement (1) holds.

Assume that $U_{c s W}^{s e_{L}} \leq A, A$ is non-decreasing in its second variable and left arbitrary $\vee$-distributive. Then, $A_{l a}=A$, $I_{A}^{L} \leq I_{U_{c s W}^{s L_{W}}}^{L}, \quad I_{A}^{L}$ is non-increasing in its first variable by Definition 3.2 and right arbitrary $\vee$-distributive by Theorem 4.6 in [8], $\left(I_{A}^{L}\right)_{u i}=I_{A}^{L}$ by Theorem 3.1(4),

$$
\begin{aligned}
& A\left(I_{A}^{L}(x, y), x\right)=A(\vee\{z \in L \mid A(z, x) \leq y\}, x) \\
& =\vee\{A(z, x) \mid A(z, x) \leq y\} \leq y \forall x, y \in L .
\end{aligned}
$$

By the virtue of Theorem 2.3(2), we see that

$$
\begin{aligned}
& (A]_{c S}^{s e_{L}}\left(\left[I_{A}^{L}\right)_{I}^{o p e_{L}}(x, y), x\right) \\
& =U_{c s M}^{e_{L}}\left(\left[I_{A}^{L}\right)_{I}^{o p e_{L}}(x, y), x\right) \wedge A\left(\left[I_{A}^{L}\right)_{I}^{o p e_{L}}(x, y), x\right) .
\end{aligned}
$$

By Example 3.1 and Theorem 3.3(1), we know that

$$
\begin{gathered}
{\left[I_{A}^{L}\right)_{I}^{\text {ope } e_{L}}(x, y)=I_{U_{c s M}^{L}}^{L}(x, y) \vee I_{A}^{L}(x, y)} \\
=\left\{\begin{array}{cc}
1 & \text { if } x=0 \text { or } y=1, \\
e_{L} \vee I_{A}^{L}(x, y) & \text { if } 0<x \leq y<1, \\
I_{A}^{L}(x, y) & \text { otherwise. }
\end{array}\right.
\end{gathered}
$$

Thus,

$$
\begin{aligned}
& (A]_{c s}^{s e_{L}}\left(\left[I_{A}^{L}\right)_{I}^{o p e_{L}}(x, y), x\right) \\
& =\left\{\begin{array}{cl}
U_{c s M}^{e_{L}}(1,0) \wedge A(1,0) & \text { if } x=0, \\
U_{c s M}^{s_{L}}(1, x) \wedge A(1, x) & \text { if } y=1, \\
U_{c s M}^{e_{L}}\left(e_{L} \vee I_{A}^{L}(x, y), x\right) \wedge A\left(e_{L} \vee I_{A}^{L}(x, y), x\right) & \text { if } 0<x \leq y<1, \\
U_{c s M}^{e_{L}}\left(I_{A}^{L}(x, y), x\right) \wedge A\left(I_{A}^{L}(x, y), x\right) & \text { otherwise. }
\end{array}\right.
\end{aligned}
$$

When $0<x \leq y<1$, noting that $I_{A}^{L}(x, y)$ and $e_{L}$ are comparable, we see that

$$
\begin{aligned}
& U_{c s M}^{e_{L}}\left(e_{L} \vee I_{A}^{L}(x, y), x\right) \wedge A\left(e_{L} \vee I_{A}^{L}(x, y), x\right) \\
& \leq \begin{cases}U_{c s M}^{e_{L}}\left(e_{L}, x\right)=x & \text { if } I_{A}^{L}(x, y) \leq e_{L}, \\
A\left(I_{A}^{L}(x, y), x\right) & \text { if } I_{A}^{L}(x, y) \leq e_{L} .\end{cases}
\end{aligned}
$$

So, when $0<x \leq y<1$,

$$
U_{c s M}^{e_{L}}\left(e_{L} \vee I_{A}^{L}(x, y), x\right) \wedge A\left(e_{L} \vee I_{A}^{L}(x, y), x\right) \leq y .
$$

Therefore, $(A]_{c s}^{s e_{L}}\left(\left[I_{A}^{L}\right)_{I}^{o p e_{L}}(x, y), x\right) \leq y$ for all $x, y \in L$, i.e., $(A]_{c s}^{s e_{L}}$ and $\left[I_{A}^{L}\right)_{I}^{o p e_{L}}$ satisfy the GMP rule.

The theorem is proved.

\section{Conclusions and Future Works}

Constructing fuzzy connectives is an interesting topic. Recently, Su et al. [16] studied the constructions of left and right semi-uninorms, and Wang et al. [19-20, 22, 24] investigated the constructions of implications and coimplications on a complete lattice. In this paper, motivated by these works, we give out the formulas for calculating the upper and lower approximation strict left (right)-conjunctive left (right) semi-uninorms of a binary operation; lay bare the formulas for calculating the upper and lower approximation implications, which satisfy the order property, of a binary operation; reveal the relationships between the upper approximation strict left (right)-conjunctive left (right) arbitrary $\vee$-distributive left (right) semi-uninorms and lower approximation right arbitrary $\wedge$-distributive implications which satisfy the order property.

In a forthcoming paper, we will further investigate the constructions of left (right) semi-uninorms and coimplications on a complete lattice.

\section{Acknowledgements}

This work is supported by Jiangsu Provincial Natural Science Foundation of China (BK20161313), Science Foundation of Yancheng Teachers University (16YCKLQ006) and the National Natural Science Foundation of China (61379064).

\section{References}

[1] J. Fodor and M. Roubens, "Fuzzy Preference Modelling and Multicriteria Decision Support", Theory and Decision Library, Series D: System Theory, Knowledge Engineering and Problem Solving, Kluwer Academic Publishers, Dordrecht, 1994.

[2] G. J. Klir and B. Yuan, "Fuzzy Sets and Fuzzy Logic, Theory and Applications", Prentice Hall, New Jersey, 1995.

[3] E. P. Klement, R. Mesiar and E. Pap, "Triangular Norms", Trends in Logic-Studia Logica Library, Vol. 8, Kluwer Academic Publishers, Dordrecht, 2000.

[4] J. Fodor and T. Keresztfalvi, "Nonstandard conjunctions and implications in fuzzy logic", International Journal of Approximate Reasoning, 12, 69-84, 1995.

[5] J. Fodor, "Srict preference relations based on weak t-norms", Fuzzy Sets and Systems, 43, 327-336, 1991.

[6] Z. D. Wang and Y. D. Yu, "Pseudo-t-norms and implication operators on a complete Brouwerian lattice", Fuzzy Sets and Systems, 132, 113-124, 2002.

[7] Y. Su and Z. D. Wang, "Pseudo-uninorms and coimplications on a complete lattice", Fuzzy Sets and Systems, 224, 53-62, 2013.

[8] Z. D. Wang and J. X. Fang, "Residual operators of left and right uninorms on a complete lattice", Fuzzy Sets and Systems, 160, 22-31, 2009.

[9] H. W. Liu, "Semi-uninorm and implications on a complete lattice", Fuzzy Sets and Systems, 191, 72-82, 2012.

[10] Y. Ouyang, "On fuzzy implications determined by aggregation operators", Information Sciences, 193, 153-162, 2012.

[11] R. R. Yager and A. Rybalov, "Uninorm aggregation operators", Fuzzy Sets and Systems, 80, 111-120, 1996. 
[12] J. Fodor, R. R. Yager and A. Rybalov, "Structure of uninorms", Internat. J. Uncertainly, Fuzziness and Knowledge-Based Systems, 5, 411-427, 1997.

[13] M. Mas, M. Monserrat and J. Torrens, "On left and right uninorms", Internat. J. Uncertainly, Fuzziness and Knowledge-Based Systems, 9, 491-507, 2001.

[14] M. Mas, M. Monserrat and J. Torrens, "On left and right uninorms on a finite chain", Fuzzy Sets and Systems, 146, 3-17, 2004.

[15] Z. D. Wang and J. X. Fang, "Residual coimplicators of left and right uninorms on a complete lattice", Fuzzy Sets and Systems, 160, 2086-2096, 2009.

[16] Y. Su, Z. D. Wang and K. M. Tang, "Left and right semi-uninorms on a complete lattice", Kybernetika, 49, 948-961, 2013.

[17] S. Jenei and F. Montagna, "A general method for constructing left-continuous t-norms", Fuzzy Sets and Systems, 136, 263-282, 2003.

[18] Z. D. Wang, "Generating pseudo-t-norms and implication operators", Fuzzy Sets and Systems, 157, 398-410, 2006.

[19] Y. Su and Z. D. Wang, "Constructing implications and coimplications on a complete lattice", Fuzzy Sets and Systems, 247, 68-80, 2014.

[20] X. Y. Hao, M. X. Niu and Z. D. Wang, "The relations between implications and left (right) semi-uninorms on a complete lattice", Internat. J. Uncertainly, Fuzziness and Knowledge-Based Systems, 23, 245-261, 2015.

[21] M. X. Niu, X. Y. Hao and Z. D. Wang, "Relations among implications, coimplications and left (right) semi-uninorms", Journal of Intelligent and Fuzzy Systems, 29, 927-938, 2015.

[22] Z. D. Wang, "Left (right) semi-uninorms and coimplications on a complete lattice", Fuzzy Sets and Systems, 287, 227-239, 2016.

[23] X. Y. Hao, M. X. Niu, Y. Wang and Z. D. Wang, "Constructing conjunctive left (right) semi-uninorms and implications satisfying the neutrality principle", Journal of Intelligent and Fuzzy Systems, 31, 1819-1829, 2016.

[24] Z. D. Wang, M. X. Niu and X. Y. Hao, "Constructions of coimplications and left (right) semi-uninorms on a complete lattice", Information Sciences, 317, 181-195, 2015.

[25] G. Birkhoff, "Lattice Theory", American Mathematical Society Colloquium Publishers, Providence, 1967.

[26] M. Baczynski and B. Jayaram, "Fuzzy Implication", Studies in Fuzziness and Soft Computing, Vol. 231, Springer, Berlin, 2008.

[27] H. Bustince, P. Burillo and F. Soria, "Automorphisms, negations and implication operators", Fuzzy Sets and Systems, 134, 209-229, 2003.

[28] B. De Baets and J. Fodor, "Residual operators of uninorms", Soft Computing, 3, 89-100, 1999. 